This study confirmed that the mortality rate of patients with SLE is higher than that of the general population. The most frequent causes of death were circulatory disease, SLE, neoplasms, nephritis and infections. Mortality was highest in patients who were female, young, and who had a disease duration of $<1$ year; however, mortality was generally increased in patients with SLE from all demographic groups. The estimates of mortality were largely consistent across countries, although within the US there was increased mortality in black or African American patients, compared with white patients. Over the duration of the study, mortality rates for patients with SLE decreased. The authors conclude that future efforts should focus on developing ways to prevent and treat the sequelae of SLE, as well as other comorbidities, particularly cardiovascular disease.

Original article Bernatsky S et al. (2006) Mortality in systemic lupus erythematosus. Arthritis Rheum 54: 2550-2557

\section{Predictors of infliximab-related infusion reactions}

Infliximab is used to treat a variety of inflammatory disorders; however, infusion reactions can occur that might necessitate discontinuation of treatment. A study in Sweden has evaluated the predictive values of baseline antinuclear antibody (ANA) status and of current methotrexate or disease-modifying antirheumatic drug therapy for the development of infusion reactions to infliximab, in patients with chronic arthritis.

The study included 213 patients with rheumatoid arthritis and 76 patients with spondyloarthropathies who were treated at a single institution between 1995 and 2005. Infliximab $3 \mathrm{mg} / \mathrm{kg}$ was given to both groups at weeks 0 , 2 and 6 , and every 8 weeks thereafter, with the option to increase dosage or shorten treatment intervals in patients with an insufficient clinical response. Baseline ANA status was assessed at infliximab initiation, and all adverse events were recorded.

Infliximab dosage was not associated with infusion reactions. In patients with rheumatoid arthritis, concomitant methotrexate therapy seemed to protect against infusion reactions to infliximab: baseline ANA positivity and absence of methotrexate treatment were independent risk factors for infusion reactions. The risk was highest in ANA-positive patients who were not receiving methotrexate. Disease-modifying antirheumatic drugs other than methotrexate had no association with infliximab infusion reactions. In patients with spondyloarthritis, there were no associations between infliximab infusion reactions and either baseline ANA positivity or absence of methotrexate treatment, but this finding should be interpreted with caution, because of the limited statistical power of the investigation.

Original article Kapetanovic MC et al. (2006) Predictors of infusion reactions during infliximab treatment in patients with arthritis. Arthritis Res Ther 8: R131

\section{Diuretics do not increase the risk of developing gout}

The hypothesis that diuretics induce gout has generally been accepted, despite a lack of evidence from published studies. A Dutch study has now found that diuretics do not increase a patient's risk of developing gout.

This case-control study included patients over the age of 35 years treated at a primary health-care center. Case patients with a first diagnosis of gout between October 1994 and October 2002 were identified $(n=70)$; all diagnoses were retrospectively reassessed. Three control patients without gout were randomly selected from the same population and matched for age and sex to each case $(n=210)$. Cases and controls were evaluated for hypertension, overall cardiovascular morbidity, specific cardiovascular diseases, and diuretic use.

During the study period, the overall incidence of hypertension and cardiovascular morbidity was $11.1 \%$ and $9.0 \%$ of participants, respectively, and $14.0 \%$ of participants used diuretics. The incidence of gout was 232 cases per 100,000 person-years. The relative risks of developing gout for patients with hypertension, cardiovascular morbidity, or diuretic use were $2.18,3.26$ and 1.56, respectively. After correcting for indications for treatment, the incidencerate ratio for gout was $0.6(95 \% \mathrm{Cl} 0.2$ to 2.0$)$, which does not support an increased risk of gout independent of these indications.

The authors conclude that there is little evidence of diuretic-induced gout in this study, and suggest that diuretics should not be 